Ecología

\title{
Coloración atípica en murciélagos: frecuencia y fenotipos en Norte y Centroamérica e islas del Caribe y nuevos casos para México y Costa Rica
}

\author{
Atypical coloration in bats: frequency and phenotypes in North and Central America, and the \\ Caribbean islands, and new cases from Mexico and Costa Rica
}

\author{
Silvia S. Zalapa ${ }^{\mathrm{a}, *}$, Sergio Guerrero ${ }^{\mathrm{a}}$, María de Lourdes Romero-Almaraz ${ }^{\mathrm{b}}$ \\ y Cornelio Sánchez-Hernández ${ }^{\mathrm{c}}$ \\ ${ }^{a}$ Centro de Estudios en Zoología, Centro Universitario de Ciencias Biológicas y Agropecuarias, Universidad de Guadalajara, $\mathrm{km} 15.5$ Carretera \\ Guadalajara-Nogales, C.P. 45110, Zapopan, Jalisco, México \\ ${ }^{\mathrm{b}}$ Domicilio particular. Escuinapa 92 bis, Col. Pedregal de Santo Domingo, C.P. 04369, Ciudad de México, México \\ ${ }^{\mathrm{c}}$ Departamento de Zoología, Instituto de Biología, Universidad Nacional Autónoma de México, Apartado postal 70-153, C.P. 04510, Ciudad de México, México
}

Recibido el 25 de junio de 2015; aceptado el 13 de noviembre de 2015

Disponible en Internet el 16 de mayo de 2016

\begin{abstract}
Resumen
El registro de murciélagos con coloración atípica se ha incrementado en los últimos años, y la terminología que se emplea para reconocer los fenotipos es confusa. Los objetivos del presente trabajo fueron: recopilar, clasificar y catalogar los casos de fenotipos atípicos para Norte y Centroamérica e islas del Caribe entre 1930 y 2014, y documentar 9 casos adicionales para México y Costa Rica. Todos los registros sumaron 317 casos de 25 especies, 17 géneros y 4 familias, provenientes de 6 países. Los ejemplares se agruparon en 4 categorías fenotípicas; la más común fue manchas blancas, con 274 registros, seguida de albino con 23, no agouti con 11 y descolorido con 4; además, 5 ejemplares tuvieron un fenotipo combinado. Los casos adicionales para México corresponden a 5 Artibeus lituratus, un Glossophaga soricina y el primer registro de Sturnira parvidens y de Nyctinomops femorosaccus. También se documentó el segundo caso para Costa Rica, Molossus sinaloae. Se desconocen las causas de la coloración atípica en los murciélagos. Se requiere de estudios genéticos y moleculares para entender su origen y el posible impacto en las poblaciones de murciélagos.

Derechos Reservados @ 2016 Universidad Nacional Autónoma de México, Instituto de Biología. Este es un artículo de acceso abierto distribuido bajo los términos de la Licencia Creative Commons CC BY-NC-ND 4.0.
\end{abstract}

Palabras clave: Albinismo; Leucismo; Melanismo

\section{Abstract}

The recording of bats with atypical coloration has been increased in the last years, and the terminology to recognize the phenotypes is confusing. The objectives of this work were: to compile, classify, and catalogue the cases of atypical phenotypes in North and Central America and the Caribbean islands between 1930 and 2014, and to report 9 additional cases in Mexico and Costa Rica. We obtained 317 cases, representing 25 species, 17 genera, and 4 families from 6 countries. The specimens were classified in 4 phenotypic categories; the most common was white spotting with 274 records, followed by albino with 23, non agouti with 11, and colorless with 4; 5 specimens had a combined phenotype. The additional cases for Mexico were 5 Artibeus lituratus, one Glossophaga soricina, and the first records for Sturnira parvidens and Nyctinomops femorosaccus. We documented the second record from Costa Rica, Molossus sinaloae. The causes and consequences of this phenomenon in bats remain unknown. Genetic and molecular studies are necessaries to understand its origin and possible impacts in bat populations.

All Rights Reserved (C) 2016 Universidad Nacional Autónoma de México, Instituto de Biología. This is an open access item distributed under the Creative Commons CC License BY-NC-ND 4.0.

Keywords: Albinism; Leucism; Melanism

\footnotetext{
* Autor para correspondencia.

Correo electrónico: sszalapah@gmail.com (S.S. Zalapa).

La revisión por pares es responsabilidad de la Universidad Nacional Autónoma de México.
} 


\section{Introducción}

En los mamíferos, el color de la piel y del pelo se debe a la melanina, un pigmento que se produce en los melanocitos a través de un proceso complejo. La formación de los melanocitos se inicia a partir de células indiferenciadas que se originan en la cresta neural y que migran hacia la capa basal de la epidermis y el folículo piloso durante el desarrollo embrionario (Hoekstra, 2006; Jackson, 1994). Estas células se diferencian como melanocitos y llevan a cabo la melanogénesis o síntesis de melanina en los melanosomas —orgánulo del melanocito_-, los cuales son transferidos a los queratinocitos (células adyacentes a los melanocitos; Bennett y Lamoreux, 2003; Lamoreux, Delmas, Laure y Bennett, 2010; Steingrímsson, Copeland y Jenkins, 2006), lo que define el color de la piel y del pelo. La melanina puede ser de 2 tipos: eumelanina, que da los tonos cafés y/o negros, o feomelanina, para los tonos rojos y amarillos (Furdon y Clark, 2003).

La formación y migración del melanocito, así como la producción, la cantidad y la distribución de la eumelanina y de la feomelanina se regulan genéticamente, por lo que cada individuo o especie puede tener un color único o variaciones de un patrón determinado (Lamoreux et al., 2010). Sin embargo, ciertas mutaciones pueden afectar alguna etapa del proceso pigmentario, por lo que algunos ejemplares no presentan el patrón de color característico de la especie. Se sabe que en ratones de laboratorio las mutaciones que afectan a estos procesos pueden ocurrir hasta en 130 genes y 1,000 alelos (Steingrímsson et al., 2006), de los cuales se han identificado 8 en otros mamíferos (Jackson, 1997). En la literatura no genética se ha generalizado el uso de los términos «albino» o «albinismo» para referirse a cualquier tipo de hipopigmentación, y los términos «piebald» o «piebaldismo» para fenotipos con manchas blancas, lo que es inapropiado, porque piebald es uno de varios genes que producen manchas blancas (Lamoreux et al., 2010) y piebaldismo es un fenotipo específico en humanos (Jackson, 1997). También el término «melanismo» para referirse a fenotipos ennegrecidos (Jackson, 1994; Nachman, Hoekstra y d'Agostino, 2003); «leucismo» para fenotipos con manchas blancas atípicas (Jehl, 1985; Veena, Thomas, Raje y Durgekar, 2011), fenotipos blancos con ojos negros (Mitchell y Church, 2002; Moore y Ouellet, 2014; Steen y Sonerud, 2012; Van Grouw, 2006) y coloraciones pálidas o descoloridas anormales (Acevedo y Aguayo, 2008; Derlindati, Cuevas, d'Ingianti y Trucco, 2013; Sage, 1962; Snyder, 1968), y «piebald» para un fenotipo con machas claras anormales (Acevedo, Torres y Aguayo-Lobo, 2009).

Específicamente en murciélagos se usan los términos: 1) albinismo, albinismo parcial, leucismo y pied, indistintamente, para referirse a individuos con todo el pelaje blanco, o más claros que el patrón normal, o con manchas blancas en algunas zonas del cuerpo (tabla 1); 2) dilución Isabel, para un fenotipo de coloración combinada con áreas grises, oscuras y nevadas (Marín-Vásquez, González-Ibarra, Gualy y Díaz, 2013); 3) silvering, para fenotipos con las puntas del pelo plateadas (Easterla y Watkins, 1968; Münzer y Kurta, 2008); 4) melanismo, para individuos oscurecidos o con manchas oscuras (Buchanan, 1985; Marín-Vásquez et al., 2013; Trapido y
Crowe, 1942), y 5) albino y albinismo, para ejemplares con ausencia total de pigmento, con el pelo y la piel de color blanco y ojos rojos (tabla 1) (Sánchez-Hernández et al., 2010; Uieda, 2000). En América, el estudio de la coloración atípica en los murciélagos se ha limitado al registro ocasional de los individuos con variaciones de color, con excepción del trabajo de Walley (1971), en el que se recopilaron los casos de albinismo y leucismo documentados entre 1940-1968, y el de Uieda (2000), quien revisó los casos conocidos de albinismo y analizó las posibles consecuencias sobre el comportamiento y la sobrevivencia de los individuos, aunque se desconocen la frecuencia, las causas, los genes y los posibles procesos pigmentarios afectados. De acuerdo con lo anterior, los objetivos del presente trabajo son: 1) recopilar los registros publicados para Norte y Centroamérica e islas del Caribe entre 1930-2014; 2) clasificar y catalogar los fenotipos atípicos compilados, y 3) documentar 9 casos adicionales de coloración atípica en murciélagos de México y Costa Rica.

\section{Materiales y métodos}

Para analizar la frecuencia y el tipo de coloración atípica en los murciélagos citados en la literatura se integró una base de datos con el nombre de la especie, la descripción del fenotipo, el país de origen, el número de catálogo, la fecha de captura y el año de publicación. Para identificar y catalogar los fenotipos de los murciélagos se usaron 4 categorías fenotípicas (CF) con base en Lamoreux et al. (2010), quienes agruparon las mutaciones que afectan la coloración en roedores de laboratorio de acuerdo con el proceso pigmentario básico afectado y describieron el fenotipo general para cada una de ellas (tabla 2). Los ejemplares adicionales con coloración atípica para México fueron capturados en los estados de Jalisco, Colima y Oaxaca. El registro de Costa Rica se capturó en la provincia de Heredia, como parte del Primer Curso Latinoamericano de acústica en murciélagos. Todos los ejemplares se capturaron con redes de niebla y fueron liberados después de registrar su información biológica básica como número de campo, medidas convencionales, sexo, edad, condición reproductiva, hora de captura y tipo de vegetación del sitio de estudio, con excepción de un Artibeus lituratus, que se depositó en la Colección Nacional de Mamíferos de la Universidad Nacional Autónoma de México, y un Sturnira parvidens, que se depositó en la Colección de Vertebrados del Centro de Estudios en Zoología, de la Universidad de Guadalajara. Para determinar las especies se consultó literatura especializada (Álvarez, Álvarez-Castañeda y López-Vidal, 1994; Davis, 1970; Hall, 1981).

\section{Resultados}

Los casos adicionales de coloración atípica para México corresponden a 5 A. lituratus, un Glossophaga soricina y los primeros registros de S. parvidens y Nyctinomops femorosaccus. Se documentó, además, un Molossus sinaloae para Costa Rica, que representó el segundo caso en ese país.

- Artibeus lituratus. Tres ejemplares fueron capturados en el estado de Jalisco, municipio de Puerto Vallarta, Área Natural 
Tabla 1

Especies de murciélagos con coloración atípica para Norte y Centroamérica e islas del Caribe de 1930-2014.

\begin{tabular}{|c|c|c|c|c|}
\hline & $\mathrm{NF}$ & $\mathrm{n}$ & País & Referencia \\
\hline \multicolumn{5}{|l|}{ CF manchas blancas } \\
\hline \multicolumn{5}{|l|}{ Molossidae } \\
\hline Molossus sinaloae & & 1 & Costa Rica & Este trabajo \\
\hline \multirow[t]{2}{*}{ Nyctinomops femorosaccus } & Pelaje blanco aberrante & 1 & EUA & Mitchell (1963) \\
\hline & & 1 & México & Este trabajo \\
\hline \multirow[t]{5}{*}{ Tadarida brasiliensis } & Coloración aberrante & 58 & EUA & Glass (1954) \\
\hline & Manchas blancas & 149 & EUA & Herreid y Davis (1960) \\
\hline & Albinismo & 3 & EUA & McCoy (1960) \\
\hline & Manchas blancas & 1 & México & Caire y Thies (1988) \\
\hline & Leucismo & 3 & México & Sánchez-Hernández et al. (2012) \\
\hline \multicolumn{5}{|l|}{ Mormoopidae } \\
\hline Pteronotus cuadridens & Leucismo & 1 & Puerto Rico & Rodríguez-Durán y Kunz (1992) \\
\hline \multicolumn{5}{|l|}{ Phyllostomidae } \\
\hline \multirow[t]{3}{*}{ Artibeus jamaicensis } & Albinismo parcial & 1 & México & Hernández-Mijangos (2009) \\
\hline & Leucismo & 3 & México & Sánchez-Hernández et al. (2012) \\
\hline & Leucismo & 1 & México & García-Morales et al. (2012) \\
\hline \multirow[t]{2}{*}{ A. lituratus } & & 3 & México & Este trabajo \\
\hline & Leucismo & 1 & México & Sánchez-Hernández et al. (2012) \\
\hline A. watsoni & Albinismo parcial & 1 & México & Hernández-Mijangos (2009) \\
\hline Carollia sowelli & Albinismo parcial & 1 & México & Hernández-Mijangos (2009) \\
\hline Dermanura phaeotis & Leucismo & 1 & Guatemala & Trujillo y Barahona (2014) \\
\hline Glossophaga soricina & & 1 & México & Este trabajo \\
\hline Macrotus waterhousii & Leucismo & 1 & México & Sánchez-Hernández et al. (2012) \\
\hline \multirow[t]{2}{*}{ Sturnira hondurensis } & Leucismo & 1 & México & García-Morales et al. (2012) \\
\hline & Leucismo & 1 & México & Sánchez-Hernández et al. (2012) \\
\hline \multicolumn{5}{|l|}{ Vespertilionidae } \\
\hline Eptesicus fuscus & Pied & 1 & EUA & Trapido y Crowe (1942) \\
\hline Myotis grisescens & Albinismo parcial & 3 & EUA & Tuttle (1961) \\
\hline \multirow[t]{4}{*}{ M. lucifugus } & Coloración inusual & 1 & Canadá & Talerico, Jung, Barclay y Melton (2008) \\
\hline & Leucismo & 2 & Canadá & Irwin et al. (2013) \\
\hline & Manchas blancas & 1 & EUA & Trapido y Crowe (1942) \\
\hline & Leucismo & 1 & EUA & Walley (1971) \\
\hline \multirow[t]{5}{*}{ M. sodalis } & Manchas Blancas & 2 & EUA & Sealander (1960) \\
\hline & Coloración Inusual & 1 & EUA & Barbour y Davis (1970) \\
\hline & Manchas Blancas & 3 & EUA & Mumford y Whitaker (1982) \\
\hline & Manchas Blancas & 1 & EUA & Caire y Thies (1988) \\
\hline & Albinismo & 19 & EUA & Brack et al. (2005) \\
\hline M. velifer & Coloración inusual & 1 & EUA & Rogers (1965) \\
\hline \multirow[t]{3}{*}{ Perimyotis subflavus } & Coloración inusual & 1 & EUA & Goslin (1947) \\
\hline & Coloración inusual & 1 & EUA & Blair (1948) \\
\hline & Albino parcial & 2 & EUA & Bures (1948) \\
\hline \multicolumn{5}{|l|}{ CF no agouti } \\
\hline Phyllostomidae & & & & \\
\hline A. lituratus & Leucismo & 1 & México & López-Wilchis y León-Galván (2012) \\
\hline Vespertilionidae & & & & \\
\hline E. fuscus & Coloración anormal & 2 & EUA & Trapido y Crowe (1942) \\
\hline M. lucifugus & Melanismo & 1 & Canadá & Buchanan (1985) \\
\hline & Melanismo & 1 & EUA & Trapido y Crowe (1942) \\
\hline & Silvering & 1 & EUA & Münzer y Kurta (2008) \\
\hline M. sodalis & Coloración inusual & 1 & EUA & Sealander (1960) \\
\hline Nycticeius humeralis & Silvering & 1 & EUA & Easterla y Watkins (1968) \\
\hline P. subflavus & Melanismo & 2 & EUA & Osgood (1938) \\
\hline & Melanismo & 1 & EUA & Trapido y Crowe (1942) \\
\hline CF albino (todo el pelaje, pi & in pigmento) & & & \\
\hline Molossidae & & & & \\
\hline M. molossus & Albino completo & 2 & Puerto Rico & Heatwole, Arroyo-Salamán y Hernández (1964) \\
\hline T. brasiliensis & Albino completo & 2 & EUA & Herreid y Davis (1960) \\
\hline & Albino verdadero & 2 & EUA & McCoy (1960) \\
\hline Mormoopidae & & & & \\
\hline Pteronotus parnellii & Color inusual & 1 & México & Sánchez, López-Forment y Gurrola (1989) \\
\hline Phyllostomidae & & & & \\
\hline A. lituratus & Albino & 1 & México & Pozo y Escobedo-Cabrera (1998) \\
\hline Desmodus rotundus & Albino & 4 & México & Sánchez-Hernández et al. (2010) \\
\hline M. waterhousii & Color inusual & 1 & México & Sánchez et al. (1989) \\
\hline
\end{tabular}


Tabla 1 (Continuación)

\begin{tabular}{|c|c|c|c|c|}
\hline & $\mathrm{NF}$ & $\mathrm{n}$ & País & Referencia \\
\hline \multicolumn{5}{|l|}{ Vespertilionidae } \\
\hline Antrozous pallidus & Albino & 1 & EUA & Setzer (1950) \\
\hline \multirow[t]{3}{*}{ M. lucifugus } & Albino verdadero & 1 & Canadá & Smith (1982) \\
\hline & Albino & 1 & EUA & Dubkin (1952) \\
\hline & Albino & 1 & EUA & Walley (1974) \\
\hline \multirow[t]{2}{*}{ M. sodalis } & Albino completo & 1 & EUA & Caire y Thies (1988) \\
\hline & Albino & 1 & EUA & Brack y Johnson (1990) \\
\hline M. velifer & Albino & 1 & EUA & Caire y Thies (1988) \\
\hline \multicolumn{5}{|c|}{ CF albino (ojos sin pigmento, piel y pelo con pigmento reducido) } \\
\hline \multicolumn{5}{|c|}{ Phyllostomidae } \\
\hline A. lituratus & & 1 & México & Este trabajo \\
\hline G. soricina & Albinismo & 1 & México & García-Morales et al. (2010) \\
\hline M. minuta & Albinismo & 1 & Costa Rica & Gamba-Ríos (2010) \\
\hline \multicolumn{5}{|l|}{ CF descolorido } \\
\hline \multicolumn{5}{|l|}{ Phyllostomidae } \\
\hline A. jamaicensis & Albinismo parcial & 1 & México & Sánchez-Hernández et al. (2010) \\
\hline A. lituratus & & 1 & México & Este trabajo \\
\hline D. phaeotis & Coloración inusual & 1 & México & Sánchez et al. (1989) \\
\hline \multicolumn{5}{|l|}{ Vespertilionidae } \\
\hline M. lucifugus & Pied & 1 & EUA & Hamilton (1930) \\
\hline \multicolumn{5}{|c|}{ CF manchas blancas-no agouti } \\
\hline \multicolumn{5}{|l|}{ Phyllostomidae } \\
\hline \multirow[t]{2}{*}{ A. lituratus } & Leucismo & 1 & México & López-Wilchis y León-Galván (2012) \\
\hline & Leucismo & 1 & México & $\begin{array}{l}\text { García-Morales, Rojas-Martínez, Ávila-Gómez } \\
\text { y Moreno (2013) }\end{array}$ \\
\hline \multicolumn{5}{|l|}{ Vespertilionidae } \\
\hline M. sodalis & Albinismo parcial & 1 & EUA & Metzger (1956) \\
\hline \multicolumn{5}{|c|}{ CF manchas blancas-descolorido } \\
\hline \multicolumn{5}{|c|}{ Phyllostomidae } \\
\hline S. parvidens & & 1 & México & Este trabajo \\
\hline \multicolumn{5}{|l|}{ Vespertilionidae } \\
\hline M. lucifugus & Pied & 1 & EUA & Trapido y Crowe (1942) \\
\hline Total & & 317 & & \\
\hline
\end{tabular}

EUA: Estados Unidos de América.

Se indica la categoría fenotípica (CF) asignada a cada ejemplar con base en la propuesta de Lamoreux et al. (2010), el fenotipo asignado en la literatura (NF) y el número de casos (n). La nomenclatura se cita de acuerdo con Ramírez-Pulido, González-Ruiz, Gardner y Arroyo-Cabrales (2014).

Protegida Estero El Salado, sitio Las Higueras, 2040'17.48” N, $-105^{\circ} 14^{\prime} 40.41^{\prime \prime} \mathrm{O}, 4 \mathrm{~m} \mathrm{snm}$. El primer ejemplar (número de campo 3,923) fue una hembra adulta inactiva capturada en bosque espinoso con remanentes de selva mediana subcaducifolia, el 20 de agosto de 2010 a las 21:00 h. El antebrazo midió
$63.8 \mathrm{~mm}$ y pesó $51 \mathrm{~g}$. Tuvo la CF albino, con el pelaje de color gamuza claro; en el rostro se apreciaron las líneas supraorbitales, los ojos fueron de color rojo, la base de las orejas, la hoja nasal, los labios y los carpos de un tono rosa; y las membranas alares y el uropatagio de color negruzco (fig. 1a). El

Tabla 2

Categorías fenotípicas con base en la propuesta de Lamoreux et al. (2010).

\begin{tabular}{|c|c|c|}
\hline Categoría fenotípica & Proceso pigmentario afectado & Fenotipo resultante $^{\mathrm{a}}$ \\
\hline Manchas blancas & $\begin{array}{l}\text { Mutaciones que afectan el desarrollo y sobrevivencia del } \\
\text { melanocito en algunas regiones del cuerpo o en todo el } \\
\text { organismo desde el nacimiento, o muerte progresiva de los } \\
\text { melanocitos después del nacimiento }\end{array}$ & $\begin{array}{l}\text { Desde el nacimiento: áreas de pelaje o piel sin pigmento } \\
\text { — manchas blancas—o todo el pelaje del organismo } \\
\text { blanco, ambos con ojos negros } \\
\text { Progresivo: áreas de pelaje o piel grisáceas o todo el pelaje } \\
\text { del organismo gris y ojos negros }\end{array}$ \\
\hline Albino & $\begin{array}{l}\text { Mutaciones que afectan el desarrollo normal } \\
\text { del melanosoma }\end{array}$ & $\begin{array}{l}\text { Todo el pelaje, piel y ojos sin pigmento. Ojos sin pigmento, } \\
\text { piel y pelo con pigmento reducido }\end{array}$ \\
\hline Descolorido & $\begin{array}{l}\text { Mutaciones que afectan el transporte normal de los } \\
\text { melanosomas desde el melanocito hacia el queratinocito }\end{array}$ & $\begin{array}{l}\text { Colores del pelaje más claro que el normal, puede ser tono } \\
\text { grisáceo o beige atípico - lo que depende del tipo de } \\
\text { pigmento-. Ojos negros. }\end{array}$ \\
\hline No agouti & $\begin{array}{l}\text { Mutaciones que afectan el intercambio en la producción } \\
\text { del tipo de pigmento (eumelanina o feomelanina) de un } \\
\text { melanosoma. En especies que presentan pelo con bandas } \\
\text { de color diferente }\end{array}$ & $\begin{array}{l}\text { Colores claros o muy oscuros anormales. La mutación } \\
\text { puede afectar solo a cierta región del pelo; por ejemplo, } \\
\text { base o punta. }\end{array}$ \\
\hline
\end{tabular}

\footnotetext{
${ }^{a}$ Estos fenotipos son los más representativos que pueden resultar en la etapa pigmentaria afectada. Para más detalles, ver Lamoreux et al. (2010).
} 

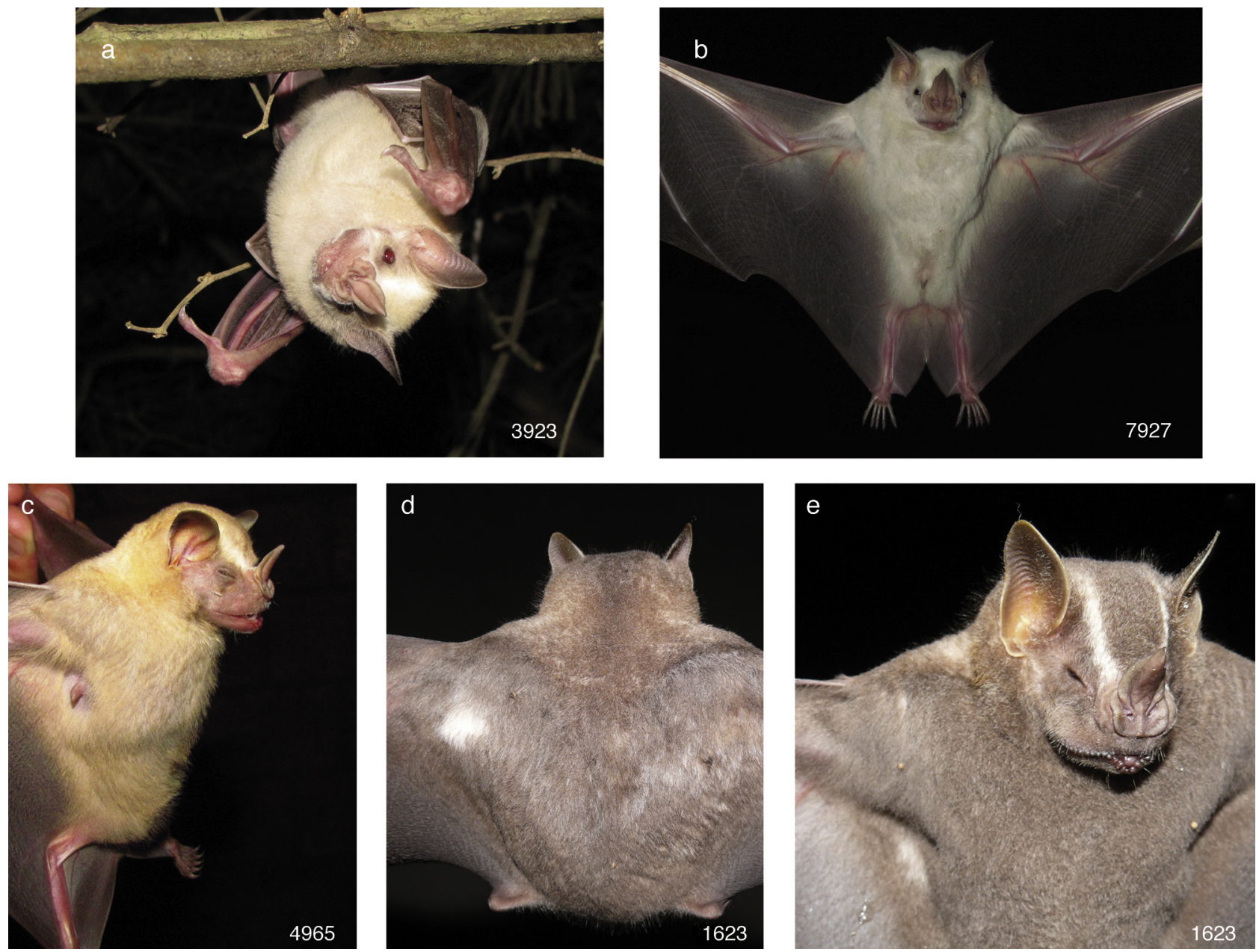

Figura 1. Artibeus lituratus: a) número 3,923, CF albino; b) número 7,927, CF manchas blancas; c) número 4,965, CF descolorido; d-e) número 1,623, CF manchas blancas, se observan en el dorso de la escápula izquierda (d) y en la parte ventral cerca a la axila derecha (e).

segundo ejemplar (número de campo 7,927) fue una hembra adulta inactiva capturada en bosque espinoso con remanentes de selva mediana subcaducifolia (29 de noviembre de 2012 a las 3:00 h). El antebrazo midió $65.6 \mathrm{~mm}$ y pesó $51 \mathrm{~g}$. Se agrupó en la CF manchas blancas, porque el pelo de la cabeza y del cuerpo fue de color blanco, la hoja nasal y la base de las orejas de color claro, mientras que las membranas alares, el uropatagio y los ojos de color normal (fig. 1b). El tercer ejemplar (número de campo 4,965) fue una hembra adulta en lactancia que se capturó en bosque espinoso con remanentes de selva mediana subcaducifolia el 7 de julio de 2011 a las 22:00 h. El antebrazo midió $68.2 \mathrm{~mm}$ y pesó $51 \mathrm{~g}$. Se asignó a la CF descolorido, porque el pelo de la cabeza y del cuerpo fue de color amarillo claro en lugar de gris o café negruzco, la hoja nasal rosa, mientras que los ojos, las orejas, las membranas alares y el uropatagio fueron de color café (fig. 1c). El cuarto murciélago (número de campo 1,623) se capturó en Jalisco, municipio de Puerto Vallarta, Área Natural Protegida Estero El Salado, sitio El Vivero, $20^{\circ} 40^{\prime} 32.76^{\prime \prime} \mathrm{N},-105^{\circ} 13^{\prime} 58.70^{\prime \prime} \mathrm{O}, 85 \mathrm{~m} \mathrm{snm}$; fue una hembra adulta inactiva con un antebrazo de $69.0 \mathrm{~mm}$ que se capturó el 4 de octubre de 2008 a las 24:00 h en bosque espinoso con palmeras de coco (Cocos nucifera). Tuvo una mancha blanca en el dorso próxima al inicio del ala izquierda (fig. 1d), en la parte ventral; otra mancha blanca cerca de la axila derecha (fig. 1e), y el resto del pelaje y de la piel fue de color normal, por lo que se agrupó en la CF manchas blancas. El quinto ejemplar (CNMA 47395) se capturó en Colima, municipio de Manzanillo, Agua Blanca, 19 $3^{\circ} 57.66^{\prime \prime} \mathrm{N},-104^{\circ} 3^{\prime} 9.12^{\prime \prime} \mathrm{O}, 141 \mathrm{~m} \mathrm{snm}$. Fue un macho adulto con testículos escrotados de $8 \times 6 \mathrm{~mm}$ y antebrazo de $64.6 \mathrm{~mm}$ y pesó $47 \mathrm{~g}$; se capturó el 9 de enero de 2002 en una red de niebla colocada en selva baja caducifolia. La coloración fue normal, excepto por una mancha blanca en la parte media del lado derecho de la espalda (fig. 2a), por lo que se agrupó en la CF manchas blancas.

- Glossophaga soricina (número de campo 8,016). Fue una hembra adulta preñada que se capturó en Jalisco, municipio de Puerto Vallarta, Área Natural Protegida Estero El Salado, sitio El Vivero, $20^{\circ} 40^{\prime} 32.76$ ” N , -105'13'58.70” O, $85 \mathrm{~m}$ snm, en bosque espinoso con árboles de mango (Mangifera indica), falsos almendros (Terminalia catappa) y guanábana (Anona muricata), el 30 de noviembre de 2012 a las 22:30 h. El antebrazo midió $36.1 \mathrm{~mm}$ y pesó $13 \mathrm{~g}$. Se asignó en la CF manchas blancas, porque tuvo un mechón de pelo de color blanco de la base de la oreja derecha a la parte media frontal (fig. 2b).

- Sturnira parvidens (CZUG 419). Fue un macho adulto con testículos escrotados capturado en Oaxaca, municipio de 

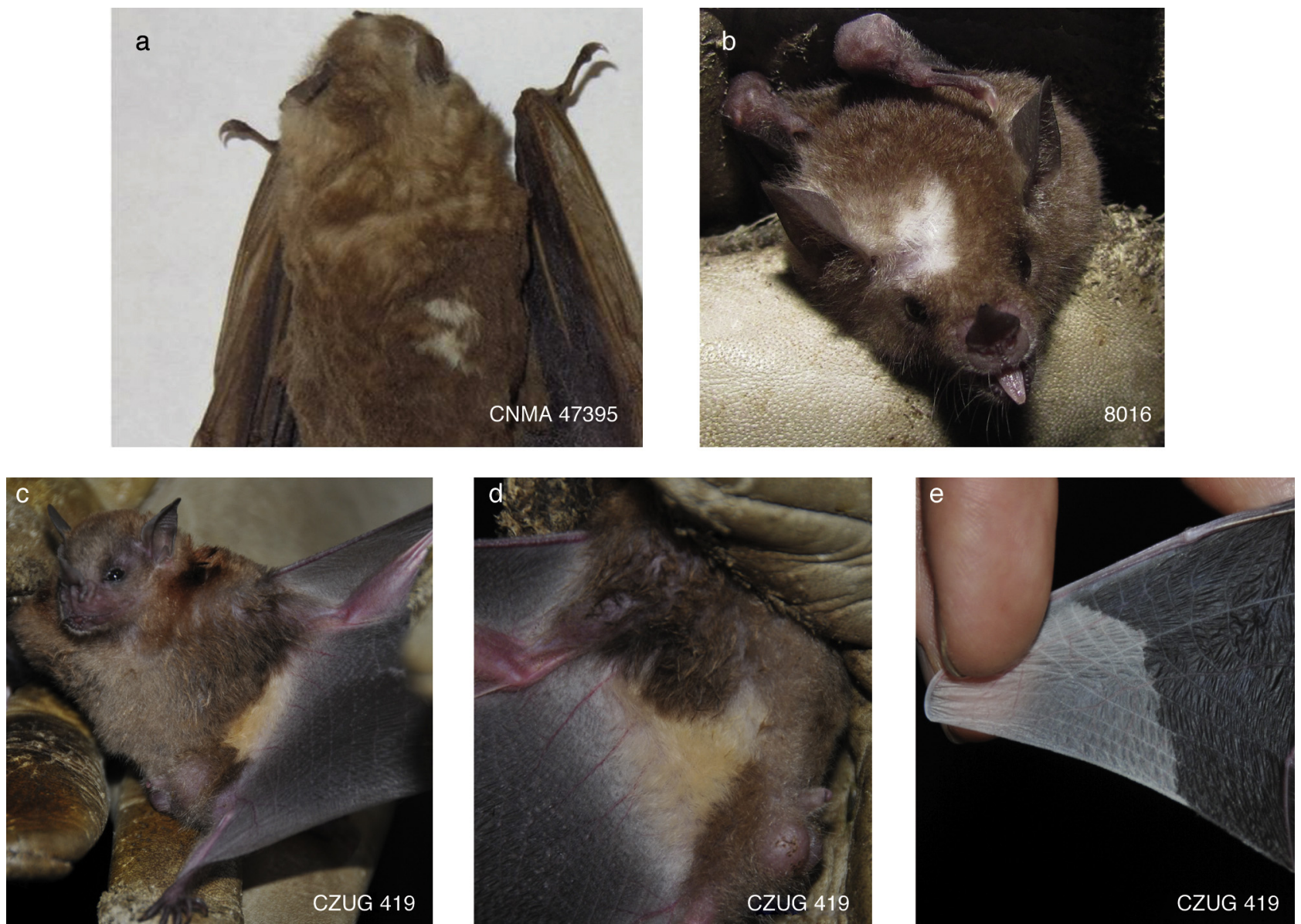

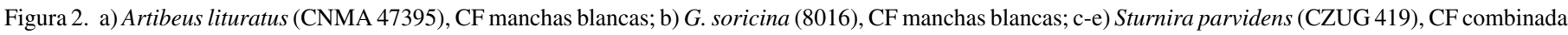
manchas blancas-descolorido, el pelo de los costados de lado ventral es amarillo (c, d) y las puntas de las 2 alas son blancas (e).

Juchitán de Zaragoza, La Venta, 16³6’30.88”N, -94'47'21.70” O, $25 \mathrm{~m} \mathrm{snm}$, en selva baja caducifolia, el 21 de abril de 2011 a las $22: 15$ h. El antebrazo midió $39.2 \mathrm{~mm}$ y pesó $19.5 \mathrm{~g}$. Se asignó a una CF combinada manchas blancas-descolorido, porque el pelo de los costados (fig. 2c) del lado ventral (fig. 2d) fue amarillo (CF descolorido), el resto del cuerpo y las membranas alares de color normal, pero las puntas de las 2 alas fueron de color blanco (CF manchas blancas; fig. 2e).

- Nyctinomops femorosaccus (número de campo 5,358). Fue un macho adulto inactivo que se capturó en Jalisco, municipio de Zapopan, Área de Protección de Flora y Fauna La Primavera, $20^{\circ} 39^{\prime} 10.03$ ' N, 103 31'28.14' O, 1,849 m snm, en una red de niebla colocada al borde de una poza en bosque de encino-pino, el 9 de diciembre de 2010 a las 19:30h. El antebrazo midió $46.5 \mathrm{~mm}$ y pesó $15 \mathrm{~g}$. La CF fue manchas blancas porque presentó un collar blanco, mientras que el resto del pelaje fue de color normal (fig. 3a).

- Molossus sinaloae. El ejemplar fue un macho subadulto con testículos abdominales que se capturó en una construcción en Costa Rica, provincia de Heredia, Reserva Ecológica La Tirimbina, Sarapiquí, $10^{\circ} 23^{\prime} 52.29^{\prime}$ N , -847'4.07' O, 48 m snm, en bosque tropical húmedo, el 8 de agosto de 2013. El antebrazo midió $49.0 \mathrm{~mm}$ y pesó $28.5 \mathrm{~g}$. Se asignó a la CF manchas blancas porque tuvo una mancha pequeña de color blanco en el pecho del lado derecho y el resto del pelaje fue de color normal (fig. 3b).

Al considerar los casos de murciélagos con coloración atípica para Norte y Centroamérica y las islas del Caribe citados en la literatura entre 1930-2014 y los documentados en este trabajo, se tienen 317 registros que se agruparon en 4 familias, 17 géneros y 25 especies. La familia con el mayor número de especies fue Phyllostomidae, con 11, seguida de Vespertilionidae, con 8, Molossidae, con 4, y Mormoopidae, con 2. La especie con el mayor número de registros fue Tadarida brasiliensis, con 218 ejemplares, seguida de Myotis sodalis, con 30, y Myotis lucifugus, con 13 (tabla 1). Los ejemplares con coloración atípica provinieron de 6 países: México tiene el mayor número de especies, 13 (36 casos); Estados Unidos de América, 10 especies (270 casos); Puerto Rico, 2 especies (3 casos); Costa Rica, 2 especies ( 2 casos); Canadá, una especie (5 casos), y finalmente Guatemala, una especie (un caso) (tabla 1). El análisis de los casos por décadas muestra un registro constante a partir de 1930, con 2 especies en las décadas de 1930, 1970 y 1990; con 3 especies en la década de 1940; con 4 en la de 1950; con 5 en la de 2000; con 7 en la de 1960, y con 8 en la de 1980, así como un incremento notable en los últimos 5 años, con 13 especies entre 2010-2014. La categoría fenotípica manchas blancas fue la más frecuente, con 274 individuos de 18 especies; la $\mathrm{CF}$ albino 

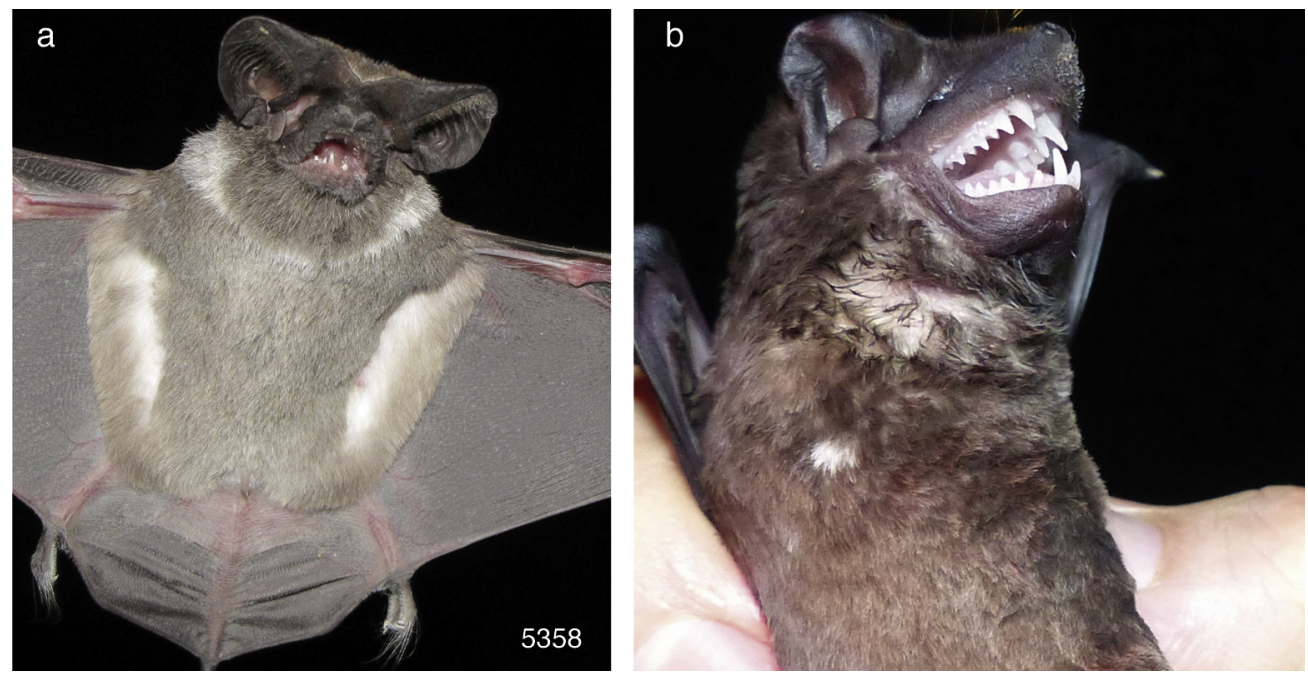

Figura 3. a) Nyctinomops femorosaccus (5358), CF manchas blancas; b) Molossus sinaloae, CF manchas blancas.

contó con 23 individuos de 12 especies; la CF no agouti, con 11 individuos de 7 especies, y la CF descolorido, con 4 individuos de 4 especies; se asignaron 3 individuos de 2 especies con una CF combinada manchas blancas-no agouti, y 2 individuos de 2 especies como CF manchas blancas-descolorido (tabla 1).

\section{Discusión}

Se considera que la frecuencia de los casos de murciélagos con coloración atípica es baja (García-Morales, GordilloChávez y Bello-Gutiérrez, 2010; Uieda, 2000), pero se ha citado que podría ser más alta de lo que se conoce (GarcíaMorales, Tejada-Duran, Ávila-Gómez, Moreno y Akmentins, 2012; Sánchez-Hernández et al., 2010). Recientemente se registró, para el Neotrópico americano, que los quirópteros son el segundo orden de mamíferos con coloración atípica, con 30 casos de 10 especies (Abreu, Machado, Barbieri, Freitas y Oliveira, 2013). Sin embargo, nosotros encontramos, por la revisión bibliográfica y los 9 ejemplares documentados en este trabajo, un total de 317 casos de 25 especies entre 1930-2014. En particular para México, los 8 nuevos registros incrementan a 36 ejemplares de 13 especies el número de casos.

Las 3 especies con el mayor número de registros fueron T. brasiliensis, $M$. sodalis y $M$. lucifugus. Cabe señalar que estas pueden formar colonias numerosas, y posiblemente cuando ocurre una mutación en el color, se transmite a otros miembros de la población. Por ejemplo, 209 de los 218 T. brasiliensis fueron registrados en 2 cuevas (Glass, 1954; Herreid y Davis, 1960), al igual que 19 de los $30 \mathrm{M}$. sodalis que se registraron en el mismo refugio (Brack, Dunlap y Johnson, 2005). Un patrón similar se encontró en las aves de Inglaterra, en donde los casos de albinismo fueron más comunes en aves altamente sociales y más sedentarias que en las aves con poblaciones aisladas y migratorias (Sage, 1963).

Por otra parte, el Área Natural Protegida el Estero El Salado, en el estado de Jalisco, fue la localidad donde se ha encontrado el mayor número de casos con diferentes fenotipos de coloración atípica (4 A. lituratus, con $3 \mathrm{CF}$ y un G. soricina), mientras que en la localidad de $1.7 \mathrm{~km} \mathrm{~S}$ y $1 \mathrm{~km} \mathrm{O}$ de Acatepec, municipio de Huautla, Hidalgo (Sánchez-Hernández et al., 2012), y la mina Andover, en Hibernia, Morris, New Jersey (Trapido y Crowe, 1942), se capturaron 2 especies con variaciones en el color. El incremento del registro de individuos con coloración atípica en los últimos años se debe posiblemente a un aumento en el esfuerzo de captura y al interés por registrar los casos. Pero podría deberse también a la presencia de contaminantes en las zonas de estudio que favorecieran el desarrollo de mutaciones fenotípicas, como se ha relacionado en golondrinas de la especie Hirundo rustica en Chernóbil, en donde los casos de albinismo se incrementaron después de la explosión del reactor (Moller y Mousseau, 2001), así como a la condición heredable dominante o semidominante de algunas mutaciones, como es el caso de White Spotting (gen KIT), que puede producir el fenotipo de manchas blancas y el de coloración generalizada blanca en ratones de laboratorio (Barsh, 2001), o a una alta endogamia en la población, en donde mutaciones de tipo recesivas como las que producen el fenotipo albino (Summers, 2009) incrementen su frecuencia.

En general, los 317 casos con coloración atípica registrados entre 1930-2014 se pudieron asignar a una de las 4 categorías fenotípicas propuestas por Lamoreux et al. (2010), lo que indica que en murciélagos ocurren mutaciones que afectan las etapas básicas del proceso pigmentario. Cabe señalar que en algunos casos fue difícil asignar el registro a alguna $\mathrm{CF}$, a pesar de la descripción o la imagen en las publicaciones revisadas, y se les asignó la categoría más afín. Además, se estableció la condición combinada, porque algunos ejemplares presentan más de una CF; por ejemplo, el $S$. parvidens de Oaxaca citado en este trabajo tiene las puntas de las alas de color blanco y una zona del pelo descolorido, patrón similar al de un $M$. lucifugus de Estados Unidos de América que presentó una mancha blanca en un ala y el pelo y el resto del cuerpo descolorido (Trapido y Crowe, 1942).

Es posible que si se revisara cada uno de los ejemplares referidos en la tabla 1 la CF asignada para algunos de ellos pudiera cambiar, debido a que en varios trabajos el fenotipo solo se 
describe como leucismo o albinismo y la descripción es muy pobre. Nuestra intención, sin embargo, es hacer una propuesta para asignar una categoría específica a un fenotipo atípico determinado o conocido y un posible proceso pigmentario afectado, para facilitar el análisis de frecuencia.

El estudio de la pigmentación en vertebrados ha permitido conocer su importancia adaptativa y puede contribuir a identificar los mecanismos biológicos que intervienen (Hoekstra, 2006), por lo que es importante, además de conocer la frecuencia, abordar el aspecto molecular y genético de este fenómeno en murciélagos con el fin de identificar las posibles causas y efectos de la presencia de coloraciones atípicas en sus poblaciones. Por ello es importante, de manera adicional al registro de la información del ejemplar, conservar al menos 2 biopsias de piel preservadas en etanol al $100 \%$ o en ultracongelador para contar con un banco de material genético que permita realizar en un futuro estudios detallados de este fenómeno.

\section{Agradecimientos}

A los estudiantes del Centro de Estudios en Zoología de la Universidad de Guadalajara por su participación en el trabajo de campo. A Thomas Sattler por su autorización para documentar el registro de $M$. sinaloae, así como a los organizadores del Primer Curso Latinoamericano de acústica en murciélagos por la captura del individuo. A J.L. Navarrete-Heredia, S.B. González-Pérez, L.M. Alcántara-Quintana y a 3 revisores anónimos por sus valiosos comentarios al manuscrito. Los ejemplares de México forman parte del proyecto «Fauna Urbana y Periurbana de Jalisco: Diversidad y Ecología» (Fideicomiso para la Protección del Estero El Salado; Universidad de Guadalajara; PROMEP UGD-CA-51). El permiso de Colector Científico fue otorgado por el Instituto Nacional de Ecología, Dirección General de Vida Silvestre: Permiso especial 06167 y 01849 (SSZH) y FAUT0103 (CS-H).

\section{Referencias}

Abreu, M. S. L., Machado, R., Barbieri, F., Freitas, N. S. y Oliveira, L. R. (2013) Anomalous colour in neotropical mammals: a review with new records for Didelphis sp. (Didelphidae, Didelphimorphia) and Arctocephalus australis (Otariidae, Carnivora). Brazilian Journal of Biology, 73, 185-194.

Acevedo, J. y Aguayo, M. (2008). Leucistic South American sea lion in Chile, with a review of anomalously color in otariids. Revista de Biología Marina y Oceanografía, 43, 413-417.

Acevedo, J., Torres, D. y Aguayo-Lobo, A. (2009). Rare piebald and partially leucistic Antarctic fur seals, Artocephalus gazella, at Cape Shirreff, Livingston Island, Antarctica. Polar Biology, 32, 41-45.

Álvarez, T., Álvarez-Castañeda, S. T. y López-Vidal, J. C. (1994). Claves para murciélagos mexicanos. La Paz, Baja California Sur: Centro de Investigaciones Biológicas del Noreste, S.C. y Escuela Nacional de Ciencias Biológicas, Instituto Politécnico Nacional.

Barbour, R. W. y Davis, W. H. (1970). Bats of America. Lexington, Kentucky: University Press of Kentucky.

Barsh, G. S. (2001). Coat color mutations, animals. Academic Press. http://dx.doi.org/10.1006/rwgn.2001.0234 [consultado 13 Jun 2015]. Disponible en: http://www.chincare.com/Pages/PGdocs/mutationsgenetics.pdf

Bennett, D. C. y Lamoreux, M. L. (2003). The color loci of mice-a genetic century. Pigment Cell Research, 16, 333-344.
Blair, W. F. (1948). A color pattern aberration in Pipistrellus subflavus subflavus. Journal of Mammalogy, 29, 178-189.

Brack, V., Jr. y Johnson, S. A. (1990). An albino Myotis sodalis. Bat Research News, 31, 8

Brack, V., Jr., Dunlap, R. K. y Johnson, S. A. (2005). Albinism in the Indiana bat Myotis sodalis. Bat Research News, 46, 55-58.

Buchanan, G. D. (1985). Comments on frequency of melanism in Myotis lucifugus. Journal of Mammalogy, 66, 178

Bures, J. A. (1948). Mammals of a limited area in Maryland. Maryland Naturalist, 18, 59-68.

Caire, W. y Thies, M. (1988). Notes on the occurrence of morphological and color aberrations in bats from Oklahoma, Missouri, and Mexico. Proceedings of the Oklahoma Academy of Science, 68, 75-76.

Davis, W. B. (1970). The large fruit bats (genus Artibeus) of Middle America, with a review of the Artibeus jamaicensis complex. Journal of Mammalogy, $51,105-122$.

Derlindati, E. J., Cuevas, M. A., d'Ingianti, S. y Trucco, C. E. (2013). First leucistic guanaco (Lama guanicoe) in the Andes Mountains of northwestern Argentina: with comments on long-term conservation implications. Southwestern Naturalist, 58, 109-111.

Dubkin, L. (1952). The white lady. London: McMillan and Co.

Easterla, D. A. y Watkins, L. C. (1968). An aberrant evening bat. Southwestern Naturalist, 13, 447-448.

Furdon, S. A. y Clark, D. A. (2003). Scalp hair characteristics in the newborn infant. Advances in Neonatal Care, 3, 286-296.

Gamba-Ríos, M. (2010). A new case of albinism in the bat Micronycteris minuta (Chiroptera: Phyllostomidae) from Costa Rica. Ecotropica, 16, 59-61.

García-Morales, R., Gordillo-Chávez, E. J. y Bello-Gutiérrez, J. (2010). Primer registro de albinismo en Glossophaga soricina (Phyllostomidae) en México. Chiroptera Neotropical, 16, 743-747.

García-Morales, R., Rojas-Martínez, A. E., Ávila-Gómez, E. S. y Moreno, C. E. (2013). Leucism in the giant fruit-eating bat (Artibeus lituratus Olfers, 1818) in the state of Hidalgo, Mexico. Chiroptera Neotropical, 19, 1212-1215.

García-Morales, R., Tejada-Durán, D., Ávila-Gómez, E. S., Moreno, C. E. y Akmentins, M. S. (2012). Registro de leucismo en Sturnira ludovici y Artibeus jamaicensis (Phyllostomidae) en México. Chiroptera Neotropical, 18, $1101-1105$

Glass, B. P. (1954). Aberrant coloration in Tadarida mexicana. American Midland Naturalist, 52, 400-402.

Goslin, R. (1947). A bat with white wing tips. Journal of Mammalogy, 28, 62.

Hall, E. R. (1981). The Mammals of North America. New York: John Wiley y Sons.

Hamilton, W. J., Jr. (1930). Notes on the mammals of Breathitt County, Kentucky. Journal of Mammalogy, 11, 306-311.

Heatwole, H., Arroyo-Salamán, J. F. y Hernández, G. (1964). Albinism in the bat, Molossus fortis. Journal of Mammalogy, 45, 476.

Hernández-Mijangos, L. A. (2009). Registros de albinismo parcial en tres especies de murciélagos filostómidos (Chiroptera: Phyllostomidae) en Chiapas, México. Chiroptera Neotropical, 15, 441-445.

Herreid, C. F. y Davis, R. B., 2nd. (1960). Frequency and placement of white fur on free-tailed bats. Journal of Mammalogy, 41, 117-119.

Hoekstra, H. E. (2006). Genetics, development and evolution of adaptive pigmentation in vertebrates. Heredity, 97, 222-234.

Irwin, A., Burns, L., Segers, J., Johnson, L., Lowe, A., Poissant, J., et al. (2013). Leucistic coloration in two little brown bats (Myotis lucifugus) in Atlantic Canada. Bat Research News, 54, 3-5.

Jackson, I. J. (1994). Molecular and developmental genetics of mouse coat color. Annual Review of Genetics, 28, 189-217.

Jackson, I. J. (1997). Homologous pigmentation mutations in human, mouse and other model organisms. Human Molecular Genetics, 6, 1613-1624.

Jehl, J. R., Jr. (1985). Leucism in eared grebes in western North America. The Condor, 87, 439-441

Lamoreux, M. L., Delmas, V., Laure, L. y Bennett, D. C. (2010). The color of mice. A model genetic network. Bryan, Texas: Wiley-Blackwell.

López-Wilchis, R. y León-Galván, M. A. (2012). A noteworthy case of leucism in Artibeus lituratus (Chiroptera: Phyllostomidae) from Oaxaca, Mexico. Chiroptera Neotropical, 18, 1111-1114. 
Marín-Vásquez, A., González-Ibarra, I., Gualy, L. y Díaz, J. D. (2013). Aberrations in coat color pattern of Colombian bats: Isabelline, leucism and melanocytes nevus. Online Journal of BioSciences and Informatics, 4, 253-265.

McCoy, C. J., Jr. (1960). Albinism in Tadarida. Journal of Mammalogy, 41, 119.

Metzger, B. (1956). Partial albinism in Myotis sodalis. Journal of Mammalogy, $37,546$.

Mitchell, H. A. (1963). Aberrant white fur in the pocketed free-tailed bat. Journal of Mammalogy, 44, 422.

Mitchell, J. C. y Church, D. R. (2002). Leucistic marbled salamanders (Ambystoma opacum) in Virginia. Banisteria, 20, 67-69.

Moller, A. P. y Mousseau, T. A. (2001). Albinism and phenotype of Barn Swallows (Hirundo rustica) from Chernobyl. Evolution, 55, 2097-2104.

Moore, J. y Ouellet, M. (2014). A review of colour phenotypes of the eastern red-backed salamander, Plethodon cinereus, in North America. Canadian Field-Naturalist, 128, 250-259.

Mumford, R. E. y Whitaker, J. O., Jr. (1982). Mammals of Indiana. Bloomington, Indiana: Indiana University Press.

Münzer, O. M. y Kurta, A. (2008). Silvering — a new color abnormality in the little brown bat, Myotis lucifugus. Bat Research News, 49, 11-12.

Nachman, M. W., Hoekstra, H. E. y d'Agostino, S. L. (2003). The genetic basis of adaptive melanism in pocket mice. Proceedings of National Academy of Sciences of the United States of America, 100, 5268-5273.

Osgood, F. L., Jr. (1938). The mammals of Vermont. Journal of Mammalogy, 19, 435-441.

Pozo, C. y Escobedo-Cabrera, J. E. (1998). Albinism in Artibeus intermedius. Bat Research News, 39, 27-28.

Ramírez-Pulido, J., González-Ruiz, N., Gardner, A. L. y Arroyo-Cabrales, J. (2014). List of recent land mammals of Mexico, 2014. Special Publications, The Museum, Texas Tech University, 63, 1-69.

Rodríguez-Durán, A. y Kunz, T. H. (1992). Pteronotus quadridens. Mammalian Species, 395, 1-4.

Rogers, G. C. (1965). Aberrant coloration in Myotis velifer incautus. Southwestern Naturalist, 10, 311.

Sage, B. L. (1962). Albinism and melanism in birds. British Birds, 55, 201-225.

Sage, B. L. (1963). The incidence of albinism and melanism in British birds. British Birds, 56, 409-416.

Sánchez, H. C., López-Forment, C. W. y Gurrola, H. M. A. (1989). Unusual coloration in three Mexican bats. Bat Research News, 30, 54-55.

Sánchez-Hernández, C., Rojas-Martínez, A., López-Vidal, J. C., ElizaldeArellano, C., Romero-Almaraz, M. L., Aguilar-López, M., et al. (2012).
Leucism in five species of bats from Mexico. Chiroptera Neotropical, 18, $1123-1127$.

Sánchez-Hernández, C., Romero-Almaraz, M. L., Taboada-Salgado, A., Almazán-Catalán, J. A., Schnell, G. D. y Sánchez-Vázquez, L. (2010). Five albino bats from Guerrero and Colima, Mexico. Chiroptera Neotropical, 16, 522-527.

Sealander, J. A., Jr. (1960). Some noteworthy records of Arkansas mammals. Journal of Mammalogy, 41, 525-526.

Setzer, H. W. (1950). Albinism in bats. Journal of Mammalogy, 31, 350.

Smith, H. G. (1982). An albino little brown bat, Myotis lucifugus from Alberta, Canada. The Canadian Field-Naturalist, 96, 217.

Snyder, D. E. (1968). A leucistic pine grosbeak. The Wilson Bulletin, 80, 333-334.

Steen, R. y Sonerud, G. A. (2012). A banc vole (Myodes glareolus) with complete leucism captured by a eurasian kestrel (Falco tinnunculus) in Norway. Annales Zoologici Fennici, 49, 306-308.

Steingrímsson, E., Copeland, N. G. y Jenkins, N. A. (2006). Mouse coat color mutations: from fancy mice to functional genomics. Developmental Dynamics, 235, 2401-2411.

Summers, C. G. (2009). Albinism: classification, clinical characteristics, and recent findings. Optometry and Vision Science, 86, 659-662.

Talerico, J. M., Jung, T. S., Barclay, R. M. R. y Melton, K. S. (2008). Abberant coloration in a little brown bat (Myotis lucifugus) from the Yukon. Northwestern Naturalist, 89, 198-200.

Trapido, H. y Crowe, P. E. (1942). Color abnormalities in three genera of northeastern cave bats. Journal of Mammalogy, 23, 303-305.

Trujillo, L. A. y Barahona, R. (2014). First record of leucism in Artibeus phaeotis (Miller, 1902) (Chiroptera: Phyllostomidae) from Guatemala. Chiroptera Neotropical, 20, 1252-1254.

Tuttle, M. D. (1961). Notes and correspondence. Bat Banding News, 2, 14-16.

Uieda, W. (2000). A review of complete albinism in bats with five new cases from Brazil. Acta Chiropterologica, 2, 97-105.

Van Grouw, H. (2006). Not every white bird is an albino: sense and nonsense about colour aberrations in birds. Dutch Birding, 28, 79-89.

Veena, S., Thomas, S., Raje, S. G. y Durgekar, R. (2011). Case of leucism in the spadenose shark, Scoliodon laticaudus (Müller and Henle, 1838) from Mangalore, Karnataka. Indian Journal of Fisheries, 58, 109-112.

Walley, H. D. (1971). A leucistic little brown bat (Myotis l. lucifugus). Transactions of the Illinois Academy of Science, 64, 196-197.

Walley, H. D. (1974). Albino little brown bat (Myotis lucifugus lucifugus) from Wisconsin, with remarks on other aberrant bats. Canadian Field-Naturalist, 88, 8081 . 